\title{
Objetos, vehículos y tripulaciones en el transporte en el Espacio Ultraterrestre
}

\author{
Objects, vehicles, and crews in outer Space \\ Transportation
}

\author{
Cristina Galdámez Ballester \\ Ingeniera aeroespacial \\ Cranfield (Bedfordshire), Reino Unido \\ crisgaldamez97@gmail.com \\ Francisca Ramón Fernández \\ Doctora en Derecho \\ Profesora titular de Derecho civil \\ Universitat Politècnica de València, España \\ frarafer@urb.upv.es
}

Estudiante en Cranfield University del Máster en Astronáutica e Ingeniería Espacial,

\section{Resumen}

El objetivo general de este trabajo es analizar las principales cuestiones legales que afectan al transporte en el Espacio Ultraterrestre. A lo largo de los diferentes puntos se estudian, el punto de vista jurídico, los distintos aspectos que conforman el transporte: la definición y tipos de objeto espacial con aportaciones en la descripción del término; tripulaciones y sus componentes. Asimismo, se plantean cuestiones relacionadas con el transporte, como el turismo y la explotación espacial. Se estudia su situación legal y viabilidad, teniendo en cuenta las peculiaridades y limitaciones que presentan. Junto a ello, se aportan ideas, con el fin de 
solucionar algunas indeterminaciones del Derecho Espacial internacional referentes al objeto de estudio.

\section{Palabras clave}

Transporte; espacio; Derecho; astronauta; objeto espacial.

\section{Abstract}

The general objective of this work is to analyze the main legal matters that affect the outer space transportation.

The legal point of view, the different aspects that are part of transportation are studied throughout the different points: the definition and the types of space object with contributions in the description of the terms, crews, and their components.

Also, transportation issues such as tourism and the space exploitation arise. Their legal situation and viability are studied considering the peculiarities and limitations they present.

Ideas are provided along with this in order to solve some indeterminacies of the international space law regarding to the object of study.

\section{Keywords}

Transport, space, law, astronaunt, space object.

Cómo citar este artículo:

Galdámez Ballester, C. y Ramón Fernández, F. (2021). Objetos, vehículos y tripulaciones en el transporte en el espacio ultraterrestre. Revista de la Facultad de Derecho y Ciencias Políticas, 51(135), pp. 368 - 395. doi: https://doi.org/10.18566/rfdcp.v51n135.a04

Recibido: 28 de noviembre de 2019

Aprobado: 19 de octubre de 2020 


\section{Introducción ${ }^{1}$}

En el presente estudio se afrontan las condiciones del régimen jurídico espacial vigente y se analizan sus fortalezas y deficiencias, enfocadas al ámbito del transporte en el Espacio Ultraterrestre, en especial atendiendo a los nuevos vehículos espaciales, teniendo en cuenta los avances tecnológicos, y los distintos problemas que se van planteando a raíz de la evolución en la normativa aplicable. En concreto, se va a examinar principalmente la legislación de ámbito internacional mediante el estudio de la Declaración de los principios jurídicos que deben regir las actividades de los Estados en la exploración y utilización del Espacio Ultraterrestre, de 1963, así como el tratado sobre los principios que deben regir las actividades de los Estados en la exploración y utilización del Espacio Ultraterrestre, incluso la Luna y otros cuerpos celestes, conocido también como Tratado del Espacio, y que se aprobó en 1967 como instrumento legislativo principal, para luego centrarnos en el acuerdo que debe regir las actividades de los Estados en la Luna y otros cuerpos celestes, de 1979, con especial atención a los preceptos aplicables al transporte que se realiza en el Espacio Ultraterrestre. Con la finalidad de dotar de una claridad expositiva al trabajo, se va a diferenciar la normativa referida que se aplica al espacio en sí, de la aplicable a los objetos espaciales y a sus tripulaciones.

Se van a definir e identificar los problemas que se pueden plantear en las distintas situaciones relacionadas con el transporte, para adoptar soluciones a las posibles carencias que se presenten.

Los principales aspectos que se desarrollan se centrarán en la aplicación de la citada normativa referenciada a los objetos espaciales, vehículos y a las tripulaciones. Del mismo modo, se mencionará el contrato de transporte espacial que afecta a empresas y entidades públicas y privadas que participen de forma indirecta en misiones espaciales. A continuación, se realiza un análisis más exhaustivo respecto de los principales componentes del transporte en el Espacio Ultraterrestre. De esta forma, se estudia el concepto de objeto espacial, y los problemas y deficiencias que este presenta, así como las diferencias con respecto al vehículo espacial.

1 Trabajo realizado en el marco del Proyecto $\mathrm{I}+\mathrm{D}+\mathrm{i}$, "Retos investigación" del programa estatal de $\mathrm{I}+\mathrm{D}+\mathrm{i}$ orientado a los retos de la sociedad del Ministerio de Ciencia, Innovación y Universidades: RTI2018-097354-B-100 (2019-2022), y del proyecto de I+D+i "Retos de Investigación", MICINN, del programa estatal de I+D+i, orientado a los retos de la sociedad (PID2019-108710RB-I00, 20202022). 
Del mismo modo, y para finalizar, se inserta un último punto de estudio donde se analizan las inminentes actividades espaciales futuras. Se plantean, en consecuencia, problemas y algunas soluciones que pueden surgir debido a las lagunas de la legislación espacial internacional actual, realizando algunas propuestas de lege ferenda que hemos considerado oportunas a efectos de una futura regulación de esta materia por parte del legislador.

\section{La regulación en el ámbito del espacio de los objetos, vehículos y tripulaciones}

\section{Algunas consideraciones}

Mientras que el derecho marítimo necesitó siglos para tomar forma, y el aéreo necesitó décadas, el Derecho Espacial Internacional se ha desarrollado de forma fugaz. Empezó a formarse en los años 60, cuando Estados Unidos empezó a lanzar asiduamente objetos al espacio (Véase más ampliamente: Cortés, 2014; Zhukov y Kolosov, 2014).

Desde entonces, distintos instrumentos normativos han ido apareciendo con la finalidad de regular las actividades espaciales de los Estados, así como la regulación en el espacio exterior, con inclusión de la Luna y otros cuerpos celestes (Lacleta, 2005), entre los que se encuentran los tratados internacionales, y los acuerdos, complementados por distintas resoluciones, siendo minoritario el Derecho interno, como en el caso de España, que no dispone de un cuerpo normativo reseñable para la regulación de las actividades espaciales, ya que una de las pocas normas nacionales que se aplican es el Real Decreto 278/1995, del 24 de febrero, por el que se crea en España el registro previsto en el convenio de 12 de noviembre de 1974 de la Asamblea General de las Naciones Unidas.

La principal legislación está constituida por tratados internacionales aplicables a la materia, siendo el más destacable el Tratado del espacio, como norma principal y que sirve de directriz a los sucesivos acuerdos y convenios que se han ido posteriormente adoptando. El Tratado del espacio, también conocido como Carta magna del espacio, fue elaborado dentro del marco de las Naciones Unidas, y en diciembre de 1966 fue aprobado con unanimidad, específicamente el 19 de diciembre de 1966 (Castro, 2001). El 27 de enero de 
1967 fue puesto a disposición de todos los Estados y el 10 de octubre de ese mismo año se hizo efectivo.

Un paso muy importante en la aprobación y desarrollo del Tratado del espacio fue la Declaración de los principios legales que gobiernan las actividades de Ios Estados en la exploración y uso del espacio exterior, que se adoptó en 1963, así como el tratado prohibitivo de la realización de pruebas de armas nucleares en la atmosfera, en el espacio exterior y bajo el agua, firmado el 5 de agosto de 1963, además del acuerdo de no transportar hasta el espacio objetos portadores de armas nucleares o cualquier otro tipo de arma de destrucción masiva (Bohinc, 2013).

Más tarde, ese mismo año, el 19 de diciembre de 1967, la Asamblea General de Naciones Unidas aprobó el Acuerdo de rescate de astronautas, de regreso de astronautas y del retorno de objetos lanzados al espacio exterior, que se dispuso para ser firmado por los estados el 22 de abril de 1968, y que se hizo efectivo el 3 de abril de ese mismo año.

El 29 de noviembre de 1971, la Asamblea General de Naciones Unidas aprobó la Convención internacional sobre riesgos por daños causados por objetos espaciales, estuvo disponible para firmar a partir del 29 de marzo de 1972 y se hizo efectiva el 30 de agosto de ese año.

En 1974 se completó la Convención del registro de objetos lanzados al espacio, la cual estuvo disponible para firmar desde el 14 de enero de 1975 y se hizo efectiva el 15 de septiembre de 1976.

Posteriormente, el 3 de julio de 1979, la Comisión del Espacio de las Naciones Unidas (denominado correctamente COPUOS, que se corresponde con Committee on the Peaceful Uses of Outer Space) finalizó el Acuerdo rector de las actividades de los Estados en la Luna y otros cuerpos celestes, el cual pudo firmarse a partir del 18 de diciembre de 1979 (Ramón, 2017).

Siendo así, los principios básicos de todas las regulaciones del espacio mencionadas antes eran aplicadas a cualquier organización bajo la condición de que declarara la aceptación de sus derechos y obligaciones, que la mayoría de los estados miembros de la organización fueran Estados parte, y que respetasen el Tratado de los principios legales que rigen las actividades en el Espacio Ultraterrestre, la Luna y los cuerpos celestes. 
Además de todos los mencionados anteriormente, muchos otros acuerdos multi o bilaterales fueron firmados, en lo que refiere a la cooperación de la exploración y uso del espacio exterior (Franco, 2014; De Faraminán, 2017), como es el caso de la Declaración sobre la cooperación internacional en la exploración y utilización del Espacio Ultraterrestre en beneficio e interés de todos los Estados, teniendo especialmente en cuenta las necesidades de los países en desarrollo (A/RES/51/122).

Otras fuentes en el ámbito del derecho espacial que surgieron posteriormente fueron el Principio relativo al sondeo remoto de la Tierra desde el espacio exterior, aprobado en 1986, y los principios relevantes al uso de energía nuclear en el espacio exterior, aprobados en 1992 (Ramón, 2017).

\section{Marco normativo sobre objetos espaciales}

Uno de los principales problemas que se han encontrado a la hora de afrontar el análisis de los objetos espaciales, ha sido precisamente la falta de concreción en su definición. Existe una falta de determinación conceptual en cuanto al término de "objeto espacial”, y ello se debe a que, en las normas estudiadas, se establecen definiciones y consideraciones referente a los objetos espaciales, pero no son unánimes, y ello provoca una confusión y falta de determinación de su concepto a la hora de establecer el régimen aplicable y considerar qué se puede entender por objeto espacial. La doctrina (Álvarez, 1992; Botero, 2013) ha incluido dentro del concepto a los cohetes espaciales, lanzadera espacial o transbordador espacial, estaciones espaciales, satélites artificiales, y sondas espaciales.

A continuación, se exponen las distintas definiciones y consideraciones legales existentes referentes a los objetos espaciales, seguidas de la correspondiente problemática debido a insuficiencia de las mismas. Por último, se plantean posibles soluciones para dichos problemas y los criterios que deberían definir un objeto espacial (Ramón, 2017).

Con esta finalidad, se diferencia el ámbito internacional aplicable a la regulación de los objetos espaciales, para después realizar un acercamiento a la regulación en España, teniendo en cuenta la escasa legislación española sobre la materia, como ya se ha señalado anteriormente.

En el ámbito internacional, resulta de especial interés indicar que, en el Tratado del espacio, de 1967, no está definido el concepto de objeto espacial. 
No obstante, en el artículo VIII del indicado texto normativo se hace referencia a la propiedad del objeto enviado al espacio, y se extiende el concepto a los objetos llevados o construidos sobre un cuerpo celeste, así como sus piezas componentes, siempre que estos se encuentren en el espacio extra-atmosférico (Zhukov et al., 2014).

En el Tratado del Espacio se observa una imprecisión y falta de definición, ya que se utilizan los términos de “objetos” o "partes componentes”, así como "instalaciones, estaciones, equipos y vehículos”, no existiendo una uniformidad en los conceptos.

Hay que indicar que el Tratado del Espacio es de obligatorio cumplimiento y tiene valor vinculante, a pesar de no haber sido firmado por todos los países, y ello, en virtud de principio de ius cogens que protege valores compartidos en el ámbito internacional (Franco, 2014).

En cuanto a los países que son partes en el tratado, según la información disponible, son 110, y 89 los que han firmado el mismo, pero no lo han ratificado (United Nations Office for Disarmament Affairs, 2020).

En el Acuerdo sobre la responsabilidad internacional por daños causados por objetos espaciales, de 1971, y en el Convenio sobre el registro de objetos lanzados al Espacio Ultraterrestre, de 1974, se refieren, en su redacción, al objeto espacial como un “objeto lanzado sobre una órbita terrestre o más allá de ella”.

Por último, en el Acuerdo Intergubernamental de 1998 (IGA), se establece, en el artículo V, que cada socio "podrá registrar como objetos espaciales los elementos de vuelo que haya suministrado”. Quedan así incluidas en la definición de objeto espacial las piezas componentes del objeto.

En los textos normativos que conciernen al Derecho Espacial Internacional aparecen referencias a vehículos y naves espaciales, y a objetos de este mismo perfil. Cuando se habla de nave espacial, se designan objetos espaciales tripulados, así como los sistemas de transporte en el espacio. Otro tipo de vehículos espaciales que deben tenerse en cuenta son estaciones orbitales o estaciones en cuerpos celestes. De este modo, se procede a distinguir entre cuatro tipos de objeto espacial, que juegan un rol específico dentro del derecho espacial: satélites artificiales terrestres, naves espaciales, estaciones orbitales y estaciones sobre cuerpos celestes (Sanz, 2005; 2007). 
En cuanto al registro de los objetos espaciales, es importante separar la regulación nacional española de la regulación en el ámbito internacional.

En el ámbito internacional, inicialmente y antes de la regulación mediante el Convenio sobre el registro de objetos lanzados al Espacio Ultraterrestre, que fue aprobado por la Asamblea General de las Naciones Unidas el 12 de noviembre de 1974, el registro internacional se basaba en una serie de datos que se presentaban voluntariamente al Secretario General de las Naciones Unidas.

Con la aprobación del convenio citado, y tras el correspondiente periodo de firma de los Estados, que se hizo efectiva el 15 de septiembre de 1976, se estableció un sistema de recogida de datos obligatorio para cada objeto lanzado a la órbita terrestre o más allá en un registro apropiado que debía ser dirigido por el estado de lanzamiento y cuya institución responsable tiene el Estado la obligación de notificar al Secretario General de las Naciones Unidas.

En el Tratado del Espacio, se refiere al registro de los objetos en su artículo VIII, estableciendo que se conservará la jurisdicción y control sobre el mismo el Estado bajo cuyo registro se encuentre el objeto.

En aplicación del Convenio sobre el registro de objetos lanzados al Espacio Ultraterrestre, en su artículo 2, cada objeto espacial solo se puede registrar una única vez, por lo que en el supuesto de que dos o más países sean responsables del lanzamiento del objeto, deberán decidir de forma conjunta cuál de los dos registrará el mismo, ya que no puede ser inscrito a la vez por los dos países.

El convenio dio comienzo a la práctica del registro internacional de los objetos espaciales. El propósito principal de dicho convenio se vincula al deseo de los Estados parte de que el secretario general de las Naciones Unidas establezca y mantenga un registro centralizado de objetos lanzados al espacio. De esta forma, se debe proporcionar, por parte del Estado, al secretario de las Naciones Unidas, una lista de datos de todos los objetos espaciales por registrar, el nombre del Estado oEstados responsables del lanzamiento, la designación apropiada para el objeto espacial o su código, la fecha, el territorio o localización de lanzamiento, los parámetros básicos de la órbita (incluyendo el periodo nodal, inclinación, apogeo y perigeo) y el propósito general del objeto espacial. Toda esta información debe ser proporcionada tan pronto como sea posible, sin llegar a estar definida una fecha límite concreta, dejándose por tanto la decisión en manos del Estado que complete el registro. 
Por otro lado, en el convenio queda indicado, que los Estados parte, y especialmente, los Estados que posean instalaciones aptas para observar y rastrear objetos espaciales, deben responder en la medida de lo posible a solicitudes de otros países miembro, o de peticiones transmitidas en su nombre, de la mano del secretario general de las Naciones Unidas, de asistencia para identificar un objeto.

\section{El Convenio sobre la responsabilidad internacional por daños causados por objetos espaciales y el registro sobre objetos espaciales como denominador común respecto al concepto del mismo}

Hay que tener en cuenta la responsabilidad por daños, ya que la reentrada de un objeto espacial en la atmósfera puede tener consecuencias en la navegación marítima y aérea y provocar daños a las personas y cosas (véase Contreras, 2014). Respecto al Convenio sobre la responsabilidad internacional por daños causados por objetos espaciales, de 1971, se vincula con el registro de objetos espaciales. El convenio contempla un régimen de responsabilidad de carácter dual, ya que determina una responsabilidad absoluta u objetiva que se aplica en los casos de daños causados en la superficie de la Tierra o a una aeronave en vuelo, y una responsabilidad de tipo subjetiva o por culpa, que se aplica a los daños causados fuera de la superficie del planeta (Gutiérrez, 1982; Ramón, 2011).

En el ámbito de la legislación española, la única regulación que contempla el objeto espacial es el Real Decreto 278/1995, del 24 de febrero, por el que se crea en España el registro previsto en el Convenio del 12 de noviembre de 1974 de la Asamblea General de las Naciones Unidas, en su artículo 4, relativo a los objetos lanzados al Espacio Ultraterrestre, hace referencia tanto a las partes componentes como al vehículo propulsor.

El Registro Nacional de Objetos Espaciales, cuyo ámbito de aplicación es el territorio español, tiene por tanto una serie de implicaciones legales como jurisdicción y control, retorno, responsabilidad internacional por daño, etc.

Debe tenerse en cuenta, que el Registro de objetos espaciales difiere sustancialmente del Registro de naves marinas y de aeronaves. Mientras que el segundo tiene como finalidad principal la identificación de los mismos, en el caso de objetos espaciales dicha identificación es necesaria en casos excepcionales (por ejemplo, un aterrizaje forzoso en territorio extranjero o daño causado por un objeto espacial en dicho territorio). Además, la 
identificación en estas situaciones suele realizarse mediante el empleo de las instalaciones terrestres nacionales encargadas del rastreo y control del objeto, no recurriendo al registro de datos.

Un ejemplo de objetos espaciales de registro nacional son los cohetes lanzadera meteorológicos o geofísicos, de los que deben indicarse datos referentes a la fecha y año de lanzamiento, tipo de cohete, coordenadas de la base de lanzamiento y el propósito del experimento. Todos estos datos se envían periódicamente a los centros internacionales de almacenamiento de datos y diseminación, donde son publicados en los catálogos de datos.

Asimismo, es necesario recordar una diferencia esencial en el registro externo y marcas identificativas de objetos espaciales con respecto a las marítimas y aéreas, cuyas marcas identificativas pueden verse a simple vista. Durante el vuelo, las marcas de identificación externas serían prácticamente indistinguibles del suelo, y el paso del objeto a través de la atmósfera durante la reentrada destruiría dichas marcas.

\section{Regulación sobre vehículos espaciales}

Aunque no están regulados detalladamente más allá de la normativa internacional correspondiente al objeto espacial, se considera interesante mencionar algunos aspectos legales con respecto a los vehículos o naves espaciales, como medios de transporte espacial (Gaggero, 2012).

La Convención del Cabo de 2001 propuso un refuerzo en la seguridad jurídica, así como una reducción del riesgo mediante el establecimiento de un mecanismo que posibilite la constitución de garantías reales sobre los bienes utilizados en la prestación de servicios espaciales, encaminadas a asegurar los créditos captados para financiar dichas actividades (Sanz, 2005; 2007).

Existe, por otro lado, el problema de los transbordadores espaciales, puesto que se trata de un objeto que presenta características de avión en el despegue, en la reinserción en la atmósfera y en el aterrizaje, y las de nave espacial en el tramo en que penetra y permanece en el Espacio Ultraterrestre. El problema es, por lo tanto, que a estos vehículos espaciales se les aplica una regulación híbrida, la legislación del derecho espacial en tanto en cuanto despegan como un cohete, y la legislación aeronáutica, ya que aterrizan como un avión. 
Un problema similar existe con las estaciones espaciales, ya que en el derecho espacial solo los objetos espaciales pueden ser matriculados de acuerdo con el Tratado de matriculación de objetos lanzados al Espacio Ultraterrestre (Ramón, 2017). Las estaciones espaciales están formadas por un conjunto de módulos de distintos países con su correspondiente matriculación, generándose una yuxtaposición ensamblada de objetos espaciales sobre la cual, como conjunto, no existe reconocimiento por parte del derecho espacial.

En el ámbito de la legislación española aplicable al territorio español, hay una falta de especificación en lo que se refiere a los vehículos espaciales, dado que, según la definición de aeronave en el artículo 11 de la Ley de Navegación Aérea del 21 de julio de 1960, se hace referencia a la capacidad de movimiento en la atmósfera.

Estos quedarían excluidos, ya que la capacidad que tienen de moverse en el espacio exterior queda excluida de las especificadas reacciones del aire.

Por lo que se refiere a las naves espaciales, la indicación de la Ley de Navegación Aérea española establece, como se ha visto, una serie de especificaciones, y se basa en la idea de movimiento y sustentación en el aire siguiendo las directrices de la Convención de París para la reglamentación de la navegación aérea, de 13 de octubre de 1919 y el Convenio sobre Aviación Civil Internacional de Chicago, de 1944. Por lo que se encuentra sujeta al registro de matrículas de aeronaves, que determina su nacionalidad. En el caso de la matriculación y el registro de las naves espaciales, las naves extranjeras tendrán la nacionalidad del Estado en el que estén matriculadas, y solo podrán ser reconocidas matrículas realizadas en un Estado distinto en caso de que la institución responsable del espacio aéreo dueño de las mismas lo permita (Zhukov et al., 2014).

La mayoría de Estados se han adherido al Convenio sobre Registro de objetos lanzados al Espacio Ultraterrestre de 1974, aunque la identificación como nave espacial debe ser independiente de la de objeto espacial, y ambas deben seguir cauces distintos.

Interesa destacar, en este punto, la futura estación internacional en órbita lunar, Space Gateway, cuyo régimen jurídico es complejo, ya que, según el acuerdo que debe regir las actividades de los Estados en la Luna y otros cuerpos celestes, de 1979, se contempla la posibilidad de que los Estados parte en el acuerdo puedan desarrollar actividades de exploración y utilización de la Luna 
en cualquier punto de su superficie o bajo la misma. Podrán, por tanto, instalar al personal y colocar los vehículos espaciales, equipo, y material en cualquier punto de la Luna.

\section{Regulaciones sobre tripulaciones en el espacio}

Hoy en día, pueden estar a cargo de una actividad espacial tanto un Estado, como una organización, ya sea gubernamental o privada, o una persona. Sin embargo, cualquiera de ellas está representada jurídicamente por la tripulación en el momento en que se estén llevando a cabo las actividades espaciales en cuestión (Farand, 2001). En el Tratado General del Espacio de 1967 quedan recogidas multitud de normas y leyes referentes al comportamiento y posición de la tripulación espacial y de los Estados parte en el tratado en el Espacio Ultraterrestre.

Es en ese mismo Tratado, cuando se perdió el carácter militar de los astronautas, y se les concedió una posición notable en el marco internacional recogida en el artículo V. Se estableció, a su vez, el principio de ayuda entre ellos.

Existen, sin embargo, otras leyes a parte del Tratado del Espacio, recogidas también en el corpus iuris spatialis, que se refieren a las normas de las actividades de los astronautas durante el desarrollo de ellas. Son destacables en esta sección el llamado Acuerdo de la Luna y el Acuerdo sobre asistencia (Sanz, 2005; 2007).

En el acuerdo que debe regir las actividades de los Estados en la Luna y otros cuerpos celestes, de 1979, que se conoce como Acuerdo de la Luna, se establece la obligación de proteger la salud y la vida de las personas que se encuentren en la Luna u otros cuerpos celestes por parte de los estados, siendo considerada toda persona que se encuentre allí astronauta y parte de la tripulación de una nave espacial. Además, los Estados quedan obligados a ofrecer refugio y ayuda si hay una persona en peligro en la Luna u otro cuerpo celeste, ya sea en otros vehículos, estaciones o equipos.

Por otra parte, en el Acuerdo sobre salvamento y devolución de astronautas y devolución de objetos lanzados al Espacio Ultraterrestre, de 1968, también llamado Acuerdo sobre asistencia, se desarrollan algunos apartados del Tratado sobre el Espacio. Indica que, en caso de encontrarse un astronauta en peligro, el Estado debe aportar todos los medios y soluciones posibles para rescatar o prestar asistencia al individuo, además de buscar la forma de devolverlo cuanto 
antes al Estado de lanzamiento. Además, declara que los demás Estados parte deberán participar y ayudar a los Estados de lanzamiento que lo soliciten.

En cuanto al astronauta como profesional, y al turista espacial, en lo relativo a la aplicación del mismo régimen jurídico o no, se discute la naturaleza del turismo espacial, ya que hay que determinar si se trata de una actividad espacial, y si el turista debe ser considerado como un pasajero o como astronauta, con la finalidad de conocer si se le deben o no aplicar los acuerdos internacionales específicos, como es el caso del Acuerdo sobre el salvamento y la devolución de astronautas y la restitución de objetos lanzados al Espacio Ultraterrestre, de 1968. Este supuesto se planteó en el supuesto de Denis Tito, que finalmente, al haber firmado una cláusula de exoneración de responsabilidad, no asumía ningún tipo de responsabilidad por parte de la nave Soyuz (Ramón, 2017).

\section{El contrato de transporte espacial}

El transporte espacial, al igual que el resto de actividades que se llevan a cabo en el Espacio Ultraterrestre, se regula por los principios establecidos en los tratados elaborados en el seno de la ONU, y principalmente por el Tratado del Espacio de 1967. Es por ello que, al transporte espacial, se le aplican todas las normas relativas a la responsabilidad por daños, registro del vehículo en el estado de lanzamiento y en el registro de las Naciones Unidas, rescate del vehículo y los astronautas, autoridad del comandante de la misión y demás normas vigentes (Sanz, 2005; 2007).

En las situaciones en las que no exista una regulación específica internacional se aplicará la legislación interna de los Estados o, en su defecto, lo establecido en el contrato de transporte, que especificará la legislación aplicable en caso de conflicto entre las partes.

A continuación, se explicarán las características especiales del contrato de transporte espacial.

En primer lugar, está el principio de los mejores esfuerzos, el cual se trata de una característica peculiar del contrato de transporte espacial.

Por otra parte, se incluye el sistema de renuncias recíprocas de responsabilidad, empleado originalmente en los contratos de transporte espacial de la NASA, aunque actualmente se reproduce en los contratos de lanzamiento efectuados prácticamente en todo el mundo. 
Además, se incluye un apartado de las disposiciones sobre el seguro espacial, complementarias al sistema de renuncias anteriormente comentado.

De manera aclaratoria, es muy importante definir el contrato de lanzamiento. Se trata básicamente de un tipo de contrato de transporte por la mayoría de conceptos. A las peculiaridades mencionadas con anterioridad, se añade el problema de que la fase de lanzamiento no está incluida jurídicamente en los textos legales.

En los contratos de transporte aparecen cuestiones relativas al transporte y los trabajos de ensamblaje e integración previos al lanzamiento en sí, que conllevan obligaciones para las partes (Hermida, 2008).

Esa preparación conjunta del lanzamiento prevista por el contrato provoca una ligera inseguridad jurídica. Sin embargo, en lo demás, se trata de un contrato de transporte convencional, aunque con unos prolegómenos de reserva de opción de lanzamiento, que se prepara con mucha antelación (en ocasiones, incluso varios años), con la finalidad de que el cohete lanzado tenga el lanzamiento y la fecha más apropiados para el usuario, condiciones que se fijan en firme al realizar el contrato de lanzamiento propiamente dicho.

Los contratos de transporte espacial son habitualmente muy similares entre sí, siguiendo el modelo que empleó la NASA en sus primeras transacciones comerciales. Constan normalmente de tres partes: la parte principal o primera, en la que se incluyen las disposiciones relativas al lanzamiento concreto; en la segunda aparecen los derechos y obligaciones de cada parte en caso de que haya que realizar un lanzamiento de reemplazo; y la tercera, que recoge las normas generales de lanzamiento.

Tras la firma del contrato, usuario y transportista asumen unas obligaciones que básicamente consisten en la realización de los mejores esfuerzos con buena voluntad para que el contrato se cumpla.

En lo que a las fechas de lanzamiento se refiere, se establece una fecha planeada que suele marcar el comienzo de la fase de lanzamiento, pero que puede cambiarse en caso de que operador o transportista pospongan 0 incluso suspendan un lanzamiento por causas justificadas. En caso de que un lanzamiento se suspenda erróneamente, la única obligación que tiene el operador es la de repetir el lanzamiento en otra fecha conveniente. 
Existe también un tipo de contrato, el de lanzamiento de entrega en órbita, que surge como respuesta a las condiciones que imponen las empresas de transporte espacial. Las condiciones para el negocio del usuario suelen ser tan desfavorables, que este queda a expensas del operador en lo que respecta a la fecha de lanzamiento.

Se trata de un contrato global, en el que se integran una serie de prestaciones hechas por la otra parte como único proveedor. Este contrato lo realiza el usuario que contrata con el fabricante del satélite su entrega, ya puesto en la órbita correspondiente. Dicha vinculación contractual se emplea principalmente por las empresas de telecomunicaciones, usuarias principales de los satélites para dicho propósito.

En caso de que el usuario prefiera un tipo de lanzador concreto para su satélite, deberá notificarlo previamente al fabricante, de modo que el satélite resulte ser compatible con el lanzador elegido. En el resto de casos, el fabricante podrá elegir el tipo de lanzador que considere conveniente. Mediante este contrato "llave en mano", el fabricante se compromete a obtener el resultado final acordado, asumiendo los riesgos correspondientes a las fases del proyecto. También, es obligación del fabricante contratar los seguros oportunos para cada una de las fases del programa, es decir, en la fabricación, así como en los periodos de pre-lanzamiento y lanzamiento.

Por otra parte, el usuario tiene la obligación de pagar el precio acordado, que será superior al de cada uno de los contratos, pero que recibirá, a cambio, el producto colocado directamente para su puesta en funcionamiento.

Este tipo de contrato está creciendo cada vez más dentro del sector espacial y es responsable del aumento de peso de los criterios comerciales por la aparición de operadores de satélite privados. Únicamente pueden realizarse con un limitado número de consorcios, multinacionales mayoritariamente, que son suministradores de sistemas espaciales completos. Por último, es importante resaltar la importancia de la elección de la base de lanzamiento para el transporte. Dicha elección tiene una gran importancia en el ámbito jurídico, ya que conlleva una serie de implicaciones en cuanto a la asignación de la jurisdicción, control y responsabilidades del objeto o vehículo espacial.

Como se ha desarrollado antes, de acuerdo con el Derecho Espacial Internacional, todos los objetos espaciales deben ser registrados por un determinado Estado de lanzamiento. Siendo así, el Estado en que se encuentre 
la base desde la que se va a hacer el lanzamiento será el Estado de jurisdicción, control y adjudicación de responsabilidades en caso de daños. Es preciso diferenciar el Estado de lanzamiento del Estado de registro, o del Estado desde el cual se realiza el lanzamiento, ya que las implicaciones en materia de responsabilidad son distintas (Molano y Rueda, 2014). El Convenio sobre la responsabilidad internacional por daños causados por objetos espaciales, de 1972, indica que el Estado de lanzamiento tendrá responsabilidad absoluta y responderá de los daños causados por un objeto espacial suyo en la superficie de la Tierra 0 a las aeronaves en vuelo (véase: Morales y Manrique, 2012; Ramón, 2017).

\section{Distintas denominaciones en el transporte en el Espacio Ultraterrestre}

\section{Objeto espacial: definición y criterios}

El concepto "objeto espacial" es el utilizado como eje a partir del que se establece la legislación que afecta tanto a vehículos, como estaciones y demás elementos lanzados al espacio. Sin embargo, como se ha explicado anteriormente, es un concepto sin definición exacta, puesto que hay tratados en que se utiliza para hacer referencia al término vehículo, mientras que se corresponde con equipo, instalación espacial.

Es por ello, es importante que en los preceptos aplicables se indique expresamente a qué elemento se está refiriendo con el fin de evitar malinterpretaciones de la legislación.

En este caso se tratará el concepto global de objeto espacial, donde se tienen en consideración todos los cohetes, satélites, naves espaciales y otros vehículos y, básicamente, cualquier artefacto construido y lanzado al espacio por el hombre, así como las partes componentes de cualquiera de ellos.

\section{Problemas del término objeto espacial}

Surge en primer lugar el problema de considerar o no las partes del propio lanzador, a pesar de que muchas de ellas no llegaran a sobrepasar la atmósfera, ni por tanto al espacio extra-atmosférico. Además, este mismo espacio carece de una delimitación y de una definición precisas. Esto conlleva, 
a su vez, a que el momento en el cual pasan a considerarse objetos espaciales no esté propiamente definido, puesto que, por ejemplo, un satélite no alcanza el estatus de objeto espacial hasta que no ha sido lanzado.

Este problema se podría solucionar con la incorporación del término “vehículo aeroespacial”, también llamado avión espacial. De este modo, durante las fases de lanzamiento, despegue y vuelo atmosférico intercontinental, así como reentrada y aterrizaje, el objeto se puede considerar como aeronave, mientras que una vez ha salido al espacio exterior y se desplaza por la órbita, pasa a ser considerado objeto espacial (Ramón, 2017).

Otros problemas son la terminología que se utiliza en referencias al objeto espacial en textos legales, la falta de referencias y calificación jurídica con respecto a la Estación Espacial Internacional (ISS) (González, 2005) debida precisamente a la falta de definición del objeto espacial, situación que debería corregirse con miras a prever la regulación general de la misma en el derecho internacional, sobre todo en los grandes tratados del derecho espacial.

\section{La basura o desechos espaciales como objetos espaciales residuales y sus efectos}

Por otro lado, otro problema que podemos destacar es el de la basura o desechos espaciales que se pueden considerar como objetos residuales como piezas o componentes que se hayan desprendido de los satélites debido al desgaste y al paso del tiempo, de objetos que han finalizado su misión, o cuya batería se ha agotado y han sido abandonados, u otros objetos perdidos durante las fases de lanzamiento o colocación en órbita (Sanz, 2005; 2007; Esquivel, 2014).

Se trata de determinar qué solución se puede adoptar para evitar los problemas de responsabilidad por caída de alguno de estos satélites o parte de los mismos, ya que muchos de ellos, ya sin combustible, cruzan constantemente el anillo geoestacionario, sin control, poniendo en peligro los otros satélites que sí están activos (Ramón, 2017).

Se indicó la posibilidad de optar por enviarlos a órbitas cementerios, utilizando los últimos reductos de combustible, ya que estas órbitas se encuentran situadas a una distancia mínima de 300 metros de la órbita geoestacionaria y no podían interceptarla (Ramón, 2017). 
Para solucionar en gran medida los problemas indicados hasta ahora, conviene establecer una serie de criterios que sirvan para definir un objeto espacial. Algunos criterios útiles son: definir la ubicación geográfica, es decir, el Espacio Ultraterrestre; establecer las características técnicas de su funcionamiento y la naturaleza de las actividades espaciales llevadas a cabo.

Además, en cuanto a los problemas de la Estación Espacial Internacional, se proponen posibles soluciones. Dado que los habitantes de su interior componen una sociedad, aunque mínima, esta deberá regirse por el derecho. En primer lugar, se debe delimitar la jurisdicción aplicable, dado que módulos de diferentes Estados dan lugar a diferentes Estados de matriculación, los cuales, de acuerdo con el artículo VIII del Tratado del espacio de 1967, conservan la jurisdicción y control sobre el objeto espacial matriculado. Esto daría lugar a una jurisdicción cuasi territorial, al igual que sucedería en un navío en alta mar, en la que se podría controlar la responsabilidad de los Estados como consecuencia de sus actividades espaciales. Por otra parte, los Estados componentes de la estación podrían llegar a establecer diferentes acuerdos en función de la autonomía de su voluntad, aunque esto conllevaría como consecuencia la aparición de conflictos jurisdiccionales.

Por último, se propone como solución para distinguir entre un objeto aeronáutico y uno aeroespacial (Sanz, 2005; 2007), sobre todo para los casos en que los lanzamientos de cuerpos al espacio realizan una parte del trayecto sobrevolando el espacio aéreo, considerar dos enfoques para definir el término “objeto espacial”, que podrían ser usados en distintos contextos mientras quede especificado cual de dichos enfoques se toma.

Por una parte, se define el objeto espacial universal, que se refiere a todo artefacto fabricado por el hombre y lanzado desde la Tierra o cualquier otro cuerpo celeste, así como sus partes componentes, cuya misión sea utilizar o explorar el espacio terrestre pacíficamente. En un satélite, por ejemplo, objeto espacial serían tanto el satélite en sí, como cada una de las partes componentes señaladas (Sanz, 2005; 2007).

Por otra, el objeto espacial funcional, que considera como objeto espacial todo aquel que es enviado al Espacio Ultraterrestre para ejercer allí una actividad. La ventaja de este enfoque es que estaría amparado en todo momento por el Derecho internacional del Espacio, sin tener en cuenta la jurisdicción de los estados jurídicos que atraviesa. 
Cabe resaltar que quedan excluidos en cualquier caso otros elementos presentes en el espacio en cuya creación no ha intervenido el hombre, como son los planetas, el polvo cósmico, cometas, etc., que serán llamados cuerpos celestes.

\section{Vehículos espaciales}

Los vehículos espaciales son considerados los porteadores en el contrato de transporte, y están incluidos según la legislación en el concepto "objeto aeroespacial”.

Actualmente, dado que las actividades espaciales comienzan a tener algunas finalidades comerciales, estas han empezado a privatizarse. Es por ello que ahora existen diversas empresas privadas orientadas al transporte en el Espacio Ultraterrestre.

En el campo del transporte espacial, deben tenerse en cuenta algunas particularidades, tales como los enormes presupuestos que se requieren para preparación desarrollo y ejecución, la cooperación, los largos períodos de tiempo necesarios para llevar a cabo una misión específica (Ramón, 2017).

Los vehículos espaciales pueden distinguirse entre sí de varias formas. A continuación, se van a describir los tipos más relevantes, comúnmente utilizados:

Por un lado, existen los vehículos de lanzamiento no recuperables, denominados internacionalmente expendable launch vehicles (ELV). Como su nombre lo indica, solo pueden ser utilizados una vez, tras la cual sus partes componentes se pierden. Están generalmente formados por la carga útil que desea transportarse, más ligera, y las etapas propulsoras. Estas últimas se desprenden y se pierden tras llevar la carga útil, también llamada cuerpo principal, hasta el Espacio Ultraterrestre. Una vez allí, el cuerpo principal utiliza pequeños propulsores para realizar los desplazamientos previstos en el espacio. Un ejemplo de ELV es el Cohete Electron, el primer cohete fabricado por la empresa Rocket Lab (Sanz, 2005; 2007).

En las misiones tripuladas, el cuerpo principal regresa a la Tierra, ya sea aterrizando o cayendo en el mar; en caso contrario, se queda en el espacio o se desintegra al atravesar la atmósfera, siendo calificados como consumibles. 
Por otro lado, existen los vehículos reutilizables (reusable launch vehicles, RLV), que se corresponden con las lanzaderas espaciales tales como el Discovery, el Challenger o el Columbia. Este grupo se remonta a 1977 y actualmente existe un artículo del Code of Federal Regulations, en el que se limita la utilización de las lanzaderas espaciales (en concreto, de la NASA) para "el transporte de cargas útiles que requieran las capacidades únicas del shuttle, que sean importantes para la seguridad nacional o para los propósitos de política exterior norteamericana” (Sanz, 2005; Sanz, 2007).

Generalmente se considera como carga útil de un vehículo de lanzamiento un satélite artificial que se debe colocar en una determinada órbita. Sin embargo, también puede incluirse en este grupo material de investigación o sondas destinadas al análisis de la Luna u otros cuerpos celestes del Sistema Solar, además de suministros para la ISS.

Recientemente, se pueden citar las nuevas naves tripuladas como la Crew Dragon, Starliner Orion, Soyuz, así como los cargueros Dragon, Cignos, Dreamchaser, HTV, Progress, ya que los Shuttles pueden ser considerados como los transbordadores iniciales, ya que la fue el transbordador Atlantis, en 2011, quien realizó la última misión del programa Shuttle.

Finalmente, se distinguen los “aviones espaciales" o vehículos aeroespaciales, aunque se encuentran actualmente en fase experimental. Se plantean como aeronaves diseñadas para comportarse en vuelo como un avión la mayor parte del tiempo, pero con la capacidad de alcanzar el espacio exterior, pudiendo realizar una órbita completa o parte de ella, consiguiendo seguidamente la reentrada al espacio aéreo y aterrizando finalmente en un aeropuerto.

Este tipo de transporte se aplicaría al denominado turismo espacial, ya que en estos momentos hay algunas empresas que ofrecen viajes orbitales, para experimentar la ausencia de gravedad, y realizar una experiencia en el Espacio Ultraterrestre (Gómez, 2008; Díaz, 2013; Rubio, 2013; Serrano y Ramón, 2013; Malpica y Nieto, 2014). Es el caso de Virgin Galactic, la empresa de Richard Branson, que ofrece viajes espaciales, tras llegar a un acuerdo con la National Aeronautics and Space Administration con el fin de establecer una colaboración con los lanzamientos de vuelos al espacio. Se realizaría a través de una dotación de cinco naves espaciales y dos nodrizas, y las naves están diseñadas para transportar a seis pasajeros y dos pilotos, alcanzando una altura de 140 kilómetros en un vuelo suborbital (Serrano y Ramón, 2013). 
También la empresa Blue Origin tiene previsto realizar vuelos suborbitales a bordo del cohete New Shepard, con una capacidad de seis pasajeros (Barragán, Bonilla, Gómez, Martínez, Moreno, Navarrete, Pérez, Villalba y Mateus, 2019).

\section{Régimen futuro en el espacio exterior: nuevas actividades espaciales}

\section{Derecho Espacial Internacional de lege ferenda}

Consideramos que los distintos problemas que hemos expuestos nos hacen reflexionar sobre cómo podría realizarse una regulación internacional que aportara una solución a los mismos. La finalidad de la exposición de este apartado es esbozar algunas propuestas, de lege ferenda, en relación con las actividades que conciernen al régimen legal del espacio exterior. Junto a ello, se va a incidir en indicar algunas de las deficiencias del régimen jurídico actualmente aplicable (Vázques, 2010; Moreno, 2013), con la finalidad de plantear posibles cambios que permitieran corregir los defectos, sobre todo en lo que respecta a definición de términos, expuestos en los apartados anteriores. Resaltar que se presenta y enfatiza el uso pacífico del espacio y la prevención de una carrera de armamentos (Sanjosé, 1990), ya que tenemos que tener en cuenta los principios que rigen el Tratado del Espacio, con lo que las actividades que se vayan a realizar en el espacio deben respetar el principio de uso pacífico, así como las limitaciones sobre la comercialización de los recursos de la Luna y los demás cuerpos celestes, siguiendo las directrices del acuerdo que debe regir las actividades de los Estados en la Luna y otros cuerpos celestes.

Siguiendo esta premisa, se expondrá un punto de vista práctico de las actividades que se pudieran adelantar en el espacio, especialmente desde la perspectiva de la explotación. Resulta evidente que los avances tecnológicos y científicos han llevado a la humanidad a un estadio donde se deben considerar los próximos pasos a seguir, con la finalidad de habilitar una explotación justa y equitativa de los recursos que podrían llegar a obtenerse del espacio exterior. No obstante, también los recursos naturales del espacio, por ejemplo, en el caso de la Luna, han sido objeto de conflicto (Prado, 2020) por la imposibilidad de ser explotados por el ser humano, motivo por el cual las grandes potencias no firmaron el denominado acuerdo Luna. 
Se van a efectuar algunas aportaciones sobre los cambios que se podrían realizar en el derecho espacial internacional con el fin de adaptarse a las actividades futuras en el espacio exterior. Se analizará brevemente el papel que desempeñará la legislación en estas actividades, así como la posible evolución de las leyes referentes al Espacio Ultraterrestre y los factores más importantes de dicho proceso evolutivo.

Ya en los años en que empezaba a aparecer la actividad espacial, muchas de las personas involucradas en la preparación del marco de referencia para el derecho espacial internacional se dieron cuenta de que tal y como se planteaba, la ley limitaba un posible desarrollo comercial del espacio exterior (Bohinc, 2013). Bien es cierto que en los años 60 parecía imposible que se colocasen satélites artificiales en órbita antes del cambio de milenio, para proporcionar servicios como teledetección, predicción climática, transmisión directa de televisión, telecomunicaciones, sistemas de posicionamiento global e incluso el habitar humano en estaciones espaciales (Bohinc, 2013). Resultaba impensable llegar a alcanzar la cantidad de satélites y objetos espaciales orbitando la Tierra que hay actualmente.

Sin embargo, ya en 1964 aparecieron especulaciones que sugerían que la exploración del Espacio Ultraterrestrey la posible comercialización no dependían únicamente de las organizaciones científicas existentes especializadas en sus campos profesionales, sino también de otras organizaciones profesionales, cuyos campos estarían conectados indirectamente con el espacio exterior. Esto afecta especialmente a los países más pequeños que, de otro modo, no pueden realizar actividades espaciales por sí mismos.

En este sentido, otro de los aspectos que interesa destacar es la utilización del Espacio, que se limita principalmente por el principio de uso pacífico. Se citan dos resoluciones de interés: la Resolución 1884 (XVIII) del 17 de octubre de 1963, denominada como la resolución "no bombas en órbita”, establece la obligación de los Estados de abstenerse de poner en órbita alrededor de la Tierra cualquier objeto portador de armas nucleares o cualquier otro tipo de armas de destrucción en masa, instalar tales armas en los cuerpos celestes, o estacionarlas en el espacio exterior en cualquier otra forma.

El Tratado del espacio, al que se hace referencia en varias ocasiones, establece que los Estados parte en el tratado se comprometen a no colocar en órbita alrededor de la Tierra ningún objeto portador de armas nucleares ni de 
ningún otro tipo de destrucción en masa, y tampoco otales armas en el Espacio Ultraterrestre en ninguna otra forma (Ramón, 2017).

Este principio de uso pacífico es uno de los pilares fundamentales y es la esencia teleológica del corpus iuris spatialis. Representa una norma de ius cogens, y es respetada por la comunidad internacional, siendo una norma imperativa y que no puede ser derogada por la voluntad particular (Ramón, 2017).

La verdadera importancia de este principio radica en la garantía de paz y seguridad, ya que el posicionamiento de objetos portadores de armas supondría una preponderancia militar de los países sobre todos los demás al poder efectuar unos actos que tendrían consecuencias devastadoras para la humanidad.

Este principio se enfrenta con el obstáculo de su falta de desarrollo normativo completo (Ramón, 2017).

La era moderna de la globalización, caracterizada por el aumento en los movimientos transfronterizos de personas, bienes y servicios, requiere la armonización y unificación de la legislación nacional respectiva. Las actividades en el espacio exterior y las relacionadas se han convertido en parte de este proceso global, y el derecho espacial internacional tendrá que adaptarse adecuadamente a las mismas. Es una realidad que el cuerpo normativo tiene carencias y una falta de actualización a la evolución de la tecnología espacial, que, además, no existe una proliferación de normas, sino que toda la normativa gira en torno al Tratado del Espacio.

Aspectos actuales como protección de datos, inteligencia artificial y nuevas tecnologías de la información y comunicación no se contemplan en la legislación del derecho espacial; es como si fuera un cuerpo normativo que padece una obsolescencia y necesita una adaptación a los nuevos derechos que se han ido conformando con la legislación en el ámbito civil y mercantil principalmente.

Hay dos factores principales que contribuyeron a la situación actual; en primer lugar, las decisiones de UN-COPUOS se toman por consenso. Por un lado, esto significa que los países tienen que formular una aceptación universal, pero, por otro lado, también da como resultado términos abstractos, vagos y limitados (falta de definiciones precisas que conducen a grandes problemas y deja espacio para especulaciones y malas interpretaciones, como ya se ha 
visto en apartados previos), ya que el resultado del COPUOS a menudo se reduce al mínimo denominador común.

En segundo lugar, el ritmo de los avances tecnológicos y los descubrimientos científicos en el sector tecnológico-espacial es mucho más rápido que el progreso del derecho espacial internacional. Esto da lugar a la aparición de nuevas preguntas que aún no han sido consideradas por el Legislador.

En este aspecto, la Declaración sobre los Beneficios Espaciales adoptada por la Asamblea General de Naciones Unidas, en 1996, juega un papel importante, ya que mejora la situación general de atraso relativo de los países en desarrollo. Sin embargo, el objetivo principal de esta declaración era establecer un marco legal que favoreciera la proliferación de la tecnología espacial, así como la distribución de los beneficios derivados de las actividades espaciales. Curiosamente, durante la redacción de esta declaración, los Estados en desarrollo suavizaron sus solicitudes y, por lo tanto, el texto final es relativamente diferente de los objetivos iniciales (Lee, 2009).

Las disposiciones constituyen un marco legal según el cual cualquier exploración y uso del Espacio Ultraterrestre debe realizarse en el interés de todos los países. Una vez más, esto reivindica la libertad de exploración y uso del espacio exterior, pero también les recuerda a los países competentes en el espacio que deben realizar sus actividades en beneficio de todos los Estados. Además, esto también propicia la cooperación internacional, a la vez que anima a las naciones que no pueden llevar a cabo misiones espaciales de forma independiente, a cooperar en proyectos conjuntos y participar así en la exploración espacial.

Además, los crecientes usos económicos de la tecnología espacial y la privatización están conduciendo a aplicaciones más amplias del Derecho Internacional Espacial privado. Estas aplicaciones tendrán que ser estudiadas y respaldadas por procedimientos legales adecuados, con el fin de evitar polémicas. En la conferencia UNISPACE III, se analizó la posibilidad de establecer un mecanismo eficiente para solucionar polémicas que surjan en relación con la comercialización del espacio (Bohinc, 2013).

Hay que indicar siguiendo a Martín (2018) que este cuerpo normativo ha quedado obsoleto, ya que no se adecua a las nuevas prácticas, parece haber alcanzado la normativa un límite, más allá del cual, no se legisla. Y ello, consideramos que es un error, ya que la obsolescencia en la que se encuentra 
actualmente el Derecho Espacial deja con numerosos interrogantes muchas de las cuestiones que hemos abordado en el presente estudio, y que posiblemente con una legislación actualizada podrían quedar resueltas.

Por último, se anhela que el espacio no sea dividido ni fraccionado, que no sea un espejo ni reflejo de lo que ha sucedido en la Tierra, en la que han imperado la conquista y el colonialismo. Anhelamos que el espacio ultraterreste sea un lugar para la humanidad trabajando en armonía para mejorar las vidas de la sociedad al explorar y posiblemente explotar los recursos espaciales para el bien común, en un espíritu de cooperación. No obstante, esta desiderata personal es difícil de mantener en un mundo actual competitivo, en el que la carrera espacial se ha convertido en un objetivo por conseguir, y los distintos países abogan por mantener más las discrepancias que la unidad orientada al bien del ser humano.

\section{Conclusiones}

La carrera espacial propulsó la creación de leyes para su regimiento. Las más destacables fueron el Tratado del Espacio de 1967, así como los distintos Acuerdos y Convenios aludidos y relacionados con el mismo. Estos instrumentos normativos conforman la base del régimen jurídico del Espacio Ultraterrestre, actualmente vigente en el ámbito internacional.

Este marco normativo, sin embargo, presenta algunas deficiencias. La más relevante se refleja en la falta de delimitación del Espacio Ultraterrestre. La mayoría de los textos legales del régimen jurídico espacial hace referencia a normas que se aplican en el Espacio Ultraterrestre.

Este, sin embargo, no está definido en ninguno de ellos. Se han elaborado distintas teorías y criterios con la finalidad de establecer los límites entre los espacios aéreo y ultraterrestre, aunque ninguna se ha impreso en ningún acuerdo de forma que resultase definitiva.

Otra deficiencia es la falta de definición en los textos reguladores del concepto “objeto espacial”. En el Tratado del Espacio se hace referencia de forma indistinta a objeto y vehículo espaciales.

En otros textos normativos a los que hemos hecho referencia, se da a entender que vehículo espacial se refiere a un objeto espacial tripulado. Se 
considera muy importante, por lo tanto, el establecimiento de una definición específica general de “objeto espacial”, así como de los distintos tipos de estos.

Respecto a las limitaciones, son destacables los problemas que surgen a la hora de definir el Estado legal del vehículo, y la autorización y registro del mismo, sobre todo en el caso del prototipo SpaceShipOne, basado en un avión que lleva anexionada una cápsula espacial que se libera a una determinada altura. Al tratarse de dos tipos de vehículos diferentes, a los que deben aplicarse dos regímenes jurídicos distintos, pueden aparecer controversias. Como se ha indicado, se propone como solución considerar el conjunto aeronave-objeto espacial como uno solo hasta el momento de la separación, y como dos distintos a partir de este momento, a la hora de aplicar la normativa existente.

Se han planteado problemas derivados de otras actividades espaciales futuras, tales como la explotación de recursos en el Espacio Ultraterrestre, así como el posible uso o utilización militar. El principal problema es que el sector tecnológico espacial se ha desarrollado a mayor velocidad que el marco legal correspondiente. Es por ello que, de nuevo, aparecen retos a la hora de plantear estas nuevas actividades. Dado que los recursos de la Tierra son limitados y se están explotando a grandes velocidades, se encuentra especialmente interesante considerar la explotación pacífica del Espacio. El problema es que, para que esta llegara a ser posible sin generar a su paso un sinfín de polémicas, es necesaria la creación de un nuevo texto legal o la modificación exhaustiva del Tratado del Espacio.

Pese a las numerosas limitaciones del Derecho Espacial Internacional, ha de reconocerse que se ha consolidado en un tiempo bastante inferior que el marítimo y el aéreo, y, pese a ello, ha resultado ser muy eficiente en las operaciones y actividades relacionadas con el Espacio Ultraterrestre hasta la fecha. Se considera que los textos legales son bastante completos, y se proponen algunas ampliaciones principalmente para dar lugar al desarrollo de nuevas actividades espaciales en un futuro cercano.

\section{Referencias}

Álvarez Hernández, J. I. (1992). Régimen jurídico de los objetos espaciales. Satélites de comunicación. Revista Europea de Derecho de la Navegación Marítima y Aeronáutica, 8, pp. 1421-1446.

Barragán Acero, V., Bonilla Arias, M. A., Gómez Arias, J., Martínez Pérez, A. G., Moreno Millán, K. N., Navarrete Acuña, V., Pérez, D., Silva Ramírez. I. M. M., Villalba Pérez, E. 
C. y Mateus Tovar, R. F. (2019). Análisis de los destinos turísticos del futuro. Punto de Vista, 10(5), pp. 1-17.

Bohinc, E. (2013). International Space Law: Legal Aspects of Exploiting Outer Space. Nova Gorica: Evropska Pravna Fakulteta V Novi Gorici, Recuperado de http://www. unaslovenia.org/sites/default/files/file/Bohinc.pdf

Botero Urrea, L. (2013). Régimen jurídico de los objetos espaciales. Revista de Derecho, Comunicaciones y Nuevas Tecnologías, 10, pp. 1-25.

Castro Villalobos, J. H. (2001). La tercera conferencia de las Naciones Unidas sobre el Espacio Ultraterrestre y el Derecho. Anuario Mexicano de Derecho Internacional, 1 pp. 98-119. Recuperado de https://revistas.juridicas.unam.mx/index.php/derechointernacional/article/view/5/4

Contreras Pasuy, G. (2014). Responsabilidad internacional por daños causados por objetos espaciales. Revista de Derecho, Comunicaciones y Nuevas Tecnologías, 11, pp. 1-17.

Cortés Robayo, L. (2014). Historia espacial: recuento histórico de su evolución y desarrollo. Revista de Derecho, Comunicaciones y Nuevas Tecnologías, 12, pp. 1-36.

De Faramiñán Gilbert, J. M. (2017). La cooperación internacional en el ámbito del Espacio Ultraterrestre. En C. Pérez González, A. Cebada Romero (Coord.), F. M. Mariño Menéndez (Dir.), Instrumentos y regímenes de cooperación internacional (pp. 427-447). Madrid: Trotta.

Díaz Rafael, G. (2013). Aspectos jurídicos sobre el turismo espacial. CIDA-E: Revista del Centro de Investigaciones y Difusión Aeronáutico-Espacial, 37, pp. 57-66.

Esquivel, M. ${ }^{a}$ de las M. (2014). Basura espacial: responsabilidad por daños. Protocolo para desastres provocados por caída de objetos espaciales o sus restos y por asteroides o meteoritos. CIDA-E: Revista del Centro de Investigaciones y Difusión Aeronáutico-Espacial, 38, pp. 67-80.

Farand A. (2001). The Code of Conduct for International Space Station Crews. ESA Bulletin, 105, pp. 64-68. Recuperado de https://www.esa.int/esapub/bulletin/ bullet105/bul105_6.pdf

Franco Mogollón, I. M. (2014). Consenso, confianza y cooperación en el derecho del Espacio Ultraterrestre. Revista de Derecho, Comunicaciones y Nuevas Tecnologías, 11, pp. 1-29. Recuperado de https://derechoytics.uniandes.edu.co/components/ com_revista/archivos/derechoytics/ytics160.pdf

Gaggero Montaner, M. (2012). Transporte espacial. Revista de Derecho Comercial, 5, pp. 153-164.

Gómez Roldán, A. (2008). Turismo espacial. Manual Formative de ACTA, 50, PP. 25-30. Recuperado de https://www.acta.es/medios/articulos/cultura_y_sociedad/050025. pdf

González Ferreiro, C. (2005). La estación espacial internacional: régimen jurídico. Madrid: Universidad Complutense de Madrid.

Gutiérrez Espada, C. (1982). Reservas propuestas en el Congreso de los Diputados al Convenio sobre Responsabilidad Internacional por daños causados por objetos espaciales. Anales de Derecho, 3, pp. 79-90. Recuperado de https://revistas.um.es/ analesderecho/article/view/83391/80421

Hermida, J. (2008). Contratos de transporte especial. CIDA-E: Revista del Centro de Investigaciones y Difusión Aeronáutico Espacial, 33, pp. 25-38. 
Lacleta Muñoz, J. M. (2005). El derecho en el Espacio Ultraterrestre. Documentos de trabajo (Real Instituto Elcano de Estudios Internacionales y Estratégicos), 18, pp. 1-18. Recuperado de https://www.almendron.com/politica/pdf/2005/spain/ spain_2271.pdf

Lee, R. J. (2009). Creating a Practical Legal Framework for the Commercial Exploitation of Mineral Resources. Perth: Murdoch University. Recuperado de https://core.ac.uk/ download/pdf/11232230.pdf

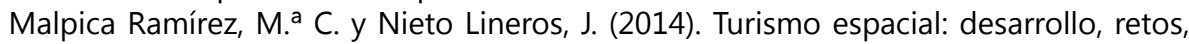
regulación jurídica y futuro. Revista de Derecho, Comunicaciones y Nuevas Tecnologías, 12, pp. 1-16.

Martín Gadea, A. (2020). El Tratado de Derecho del Espacio Ultraterrestre. Revista Electrónica de Derecho Internacional Contemporáneo, 1(1), pp. 60-64. Recuperado de https://revistas.unlp.edu.ar/Redic/article/view/9757/8573

Molano Sierra, E. y Rueda Duarte, M. A. (2014). El concepto de "Estado de lanzamiento" en el derecho del Espacio Ultraterrestre, 11, pp. 1-22.

Morales Gutiérrez, V. y Manrique Villamizar, A. Ma. (2012). Responsabilidad internacional por daños causados por objetos espaciales. Revista de Derecho, Comunicaciones y Nuevas Tecnologías, 7, pp. 1-36.

Moreno Luce, M. S. (2012). Derecho ultraterrestre. Letras jurídicas: revista de los investigadores del Instituto de Investigaciones Jurídicas U.V., 25, pp. 111-119.

Prado Alegre, E. (2020). Sobre la exploración, explotación y utilización de los recursos naturales en la Luna. Tiempo de Paz, 136, pp. 8-16.

Ramón Fernández, F. (2011). La responsabilidad civil por riesgo derivado de las actividades espaciales. En Cuestiones actuales en materia de Responsabilidad Civil (pp. 315-332). Murcia: Editum.

Ramón Fernández, F. (2017). Derecho espacial. Teoría y prácticas. Valencia: Tirant lo Blanch.

Rubio Krohne, L. (2013). Turismo espacial: aplicabilidad del acuerdo sobre salvamento y devolución de astronautas. Revista de Derecho, Comunicaciones y Nuevas Tecnologías, 10, pp. 1-22.

Sanjosé Gil, A. (1990). El principio de uso pacífico del Espacio Ultraterrestre. Tiempo de paz, 17-18, pp. 83-98.

Sanz Fernández de Córdoba, A. (2005). El transporte en el Espacio Ultraterrestre: objetos, vehículos y tripulaciones. En F. Martínez Sanz y M. V. Petit Lavall (Coord.), I Congreso Internacional de Transporte. Los retos del transporte en el siglo XXI: Castellón de la Plana, 4-6 mayo 2004, 4 (pp. 1331-1358). Valencia: Tirant lo Blanch.

Sanz Fernández de Córdoba, A. (2007). Física y derecho convergen en el Espacio Ultraterrestre. Física y Sociedad, 18, p. 39. Recuperado de http://www.cofis.es/pdf/ fys/fys18/fys_18_39.pdf

Serrano Navalón, A. I. y Ramón Fernández, F. (2013). Turismo espacial: cuestiones legales que afectan a este tipo de turismo. Revista Turismo e Sociedade, 6(3), pp. 1-25. Recuperado de https://revistas.ufpr.br/turismo/article/view/28304/20891

United Nations Office for Disarmament Affairs, 2020. Treaty on Principles Governing the Activities of States in the Exploration and Use of Outer Space, including the Moon and Other Celestial Bodies. Recuperado de http://disarmament.un.org/ treaties/t/outer_space

Zhukov, G. y Kolosov, Y. (2014). International Space Law. Moscú: Statut Publishing House. 